\title{
Adaptive detection of range-spread target in compound-Gaussian clutter without secondary data
}

\author{
Abdelmalek Mennad ${ }^{\mathrm{a}, *}$, Arezki Younsi ${ }^{\mathrm{a}}$, Mohammed Nabil El Korso ${ }^{\mathrm{b}}$, \\ Abdelhak M. Zoubir ${ }^{\mathrm{C}}$ \\ a Laboratoire Micro-Onde et Radar, Ecole Militaire Polytechnique, P.O. Box 17, 16111 Bordj El Bahri, Algeria \\ b Paris Ouest University, LEME EA4416, 50 rue de Sèvres, 92410 Ville d'Avray, France \\ c Signal Processing Group, Technische Universität Darmstadt, Merckstr. 25, 64283 Darmstadt, Germany
}

\section{Introduction}

Range-spread target detection has received an increasing attention in the last two decades [1], which is mainly due to the rise of high resolution radar (HRR). Indeed, an HRR can resolve a single target as multiple scattering centers, depending on the range extent of the target and the range resolution of the radar system [2]. The improvement of the range resolution capabilities results in a reduction of the clutter power in each range cell, hence improving the detection performance. It is worth mentioning that range-spread target detectors are useful in many situations, e.g., for the detection of large ships with coastal radars or detection of a cluster of point like target, moving at the same velocity and in close spatial proximity to each other [3].

Nevertheless, performing range-spread target detection requires to develop a proper strategy since classical adaptive detection schemes, originally proposed for point-like targets, may lead to poor performances [4,5]. This is mainly due to the fact that the target signal may contaminate the secondary cells, while these are generally assumed to be target-free in the classical context of point-like target detection.

\footnotetext{
* Corresponding author.

E-mail address: amennad@spg.tu-darmstadt.de (A. Mennad).
}

Range-spread target detection under Gaussian distributed clutter has been extensively investigated. In [6], the authors proposed a detector for spatially distributed targets embedded in white Gaussian noise, based on a priori knowledge of the target scatterer density. A modified generalized likelihood ratio test (MGLRT) detector was proposed in [7] to account for a singular estimated clutter covariance matrix. Furthermore, a fast converging detector with bounded constant false alarm rate (CFAR) property was derived in [8], under the assumption of dominant thermal noise at the receiver. On the other hand, an adaptive detection of range-spread target in homogeneous and partially homogeneous environments was addressed in [1] and [9]. Specifically, [1] and [9] assume that the secondary data are signal-free components and that they share the same covariance matrix structure, but with possibly fluctuating power.

Nevertheless, for HRR operating at low grazing angles or in a maritime environment, the Gaussian model of the clutter is no longer valid. More precisely, in [10], the clutter has been successfully modeled as a compound-Gaussian process. A compoundGaussian process is the product of a real positive random process, named texture, and a complex Gaussian process, named speckle. In the last few years, many results have been obtained for the adaptive target detection in compound-Gaussian clutter. As an example, Gini and Farina proposed the so-called generalized matched subspace detector (GMSD) [11]. The GMSD, which is a CFAR detector, aims to detect an unknown deterministic signal, but known to lie 
in a given subspace, corrupted by a compound-Gaussian clutter. In [12], the authors have derived an adaptive detector for range and Doppler distributed targets, embedded in a compound-Gaussian clutter, assuming target-free secondary cells.

In practice, the clutter covariance matrix is often unknown and commonly estimated from signal free-target secondary data in the context of compound-Gaussian process. In [13], the authors used the approximated maximum likelihood method (AML), to estimate the clutter covariance matrix, from a set of homogeneous and signal free-target secondary data. Nevertheless, to respect the nonsingularity condition of the estimated covariance matrix, a large number of secondary data is required, which in practice, can contain interfering targets or other kind of non-homogeneities [14]. To cope with the issue of low sample, structural information about the clutter covariance matrix can be exploited. Specifically, in this paper, we take advantage of modeling the clutter as an autoregressive process. Such modeling does not rely on a covariance matrix estimation step and consequently, avoids the need for large homogeneous secondary data set. This methodology has already been applied in the context of radar. More precisely, adaptive detection in unknown colored noise, modeled by an autoregressive process is addressed in [15], for known point-like target signals and in [16] for a target signal known up to a scaling factor. In [17] and [18], the authors modeled the speckle component of the clutter by a complex Gaussian autoregressive process of order $P$, for range-spread target detection in homogeneous and heterogeneous environment.

Nevertheless, in this paper, for each range cell and for an autoregressive process of order $P$ and $N$ samples, we propose to predict the $P$ first samples in a backward manner, and samples from $P+1$ until $N$ in a forward manner, using only cells under test. The proposed scheme is compared with some existing ones, especially the recent algorithms presented in [17] and [18], which use only the forward prediction, by considering just $N-P$ samples, unlike the proposed algorithm which uses all available samples. A performance analysis in terms of false alarm probability and detection probability is performed for different target models. Our numerical results indicate that the proposed detector outperforms the autoregressive GLRT (ARGLRT) detector from [17] and [18] in homogeneous and heterogeneous environment, especially when few samples are available. In addition, the proposed method outperforms the GLRT detector based on the AML estimator of the clutter covariance matrix, which requires a large number of secondary data [19].

This paper is organized as follows. In section 2, we describe the data model. In section 3 , we present the classical AR modeling and the proposed forward-backward AR modeling. Section 4 is devoted to the proposed detection algorithm. Simulation results are discussed in section 5 and finally a conclusion is given in section 6 .

\section{Problem setup}

Let us consider a radar that collects $N$ pulses during the time on target. For the $i$-th range cell, these pulses form the following complex valued received vector

$\boldsymbol{z}_{i}=\left[z_{i}(1), \ldots, z_{i}(N)\right]^{T}, i=1, \ldots, L$

where $[.]^{T}$ denotes the transpose operator and $L$ is the number of range cells under test. For sake of simplicity, we assume that the received data vectors are independent among range cells [1, 11 ] and that the target is completely contained within $L$ cells.

The detection problem is formulated as the following binary hypothesis testing problem

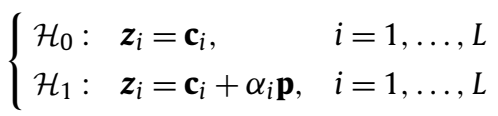

in which, $\mathbf{c}_{i}=\left[c_{i}(1), \ldots, c_{i}(N)\right]^{T}$ denotes the clutter vector, $\mathbf{p}=$ $[p(1), \ldots, p(N)]^{T}=\left[1, e^{j 2 \pi f_{d}}, \ldots, e^{j 2 \pi(N-1) f_{d}}\right]^{T}$ is the $N$-dimensional time steering vector, where $f_{d}$ denotes the normalized target Doppler frequency. In addition, we assume that $f_{d}$ is known [9, $16,18-20]$, or efficiently estimated, see e.g., [12,21]. Finally, the unknown complex amplitudes $\alpha_{i}$ are accounting for both the channel attenuation and the target cross section.

According to the compound-Gaussian model, the clutter $\mathbf{c}_{i}$ for the $i$-th range cell, is modeled as the product of two independent random variables. Specifically, $\mathbf{c}_{i}=\sqrt{\tau_{i}} \mathbf{x}$, where $\mathbf{x}$ is a $N$-dimensional zero-mean complex circular Gaussian vector, representing the speckle component, and $\tau_{i}$ denotes the variance of the underlying conditional Gaussian vector, named texture and represents the local clutter power, for each range cell. In shorthand notation, we write $\mathbf{x} \sim \mathcal{C N}(\mathbf{0}, \mathbf{M})$, where $\mathbf{M}$ is the normalized covariance matrix of the speckle, such that $\operatorname{trace}(\mathbf{M})=N$. We assume that $\tau_{i}$ and $\mathbf{x}$ are mutually independent, consequently, one has $E\left[\mathbf{c}_{i} \mathbf{c}_{i}^{H}\right]=\tau_{i} \mathbf{M}=\mathbf{M}_{c}$, with $\mathbf{M}_{c}$ denoting the clutter covariance matrix and $[.]^{H}$ indicates the conjugate transpose operator. The conditional probability density functions (pdf) of $\boldsymbol{z}_{i}$ under $\mathcal{H}_{0}$ and $\mathcal{H}_{1}$ can be expressed, respectively, as

$$
\begin{aligned}
& f\left(\boldsymbol{z}_{i} \mid \mathcal{H}_{0} ; \tau_{i}, \mathbf{M}\right)=\frac{1}{\left(\pi \tau_{i}\right)^{N}|\mathbf{M}|} \exp \left(-\frac{\boldsymbol{z}_{i}^{H} \mathbf{M}^{-1} \boldsymbol{z}_{i}}{\tau_{i}}\right) \\
& f\left(\boldsymbol{z}_{i} \mid \mathcal{H}_{1} ; \alpha_{i}, \tau_{i}, \mathbf{M}\right) \\
& \quad=\frac{1}{\left(\pi \tau_{i}\right)^{N}|\mathbf{M}|} \exp \left(-\frac{\left(\boldsymbol{z}_{i}-\alpha_{i} \mathbf{p}\right)^{H} \mathbf{M}^{-1}\left(\boldsymbol{z}_{i}-\alpha_{i} \mathbf{p}\right)}{\tau_{i}}\right)
\end{aligned}
$$

in which, $|$.$| denotes the determinant.$

\section{AR modeling}

\subsection{Forward modeling}

Let us assume that the clutter model is given by an AR process of order $P$. Then, for $n=P+1, \ldots, N$ and $i=1, \ldots, L$, we can write

$c_{i}(n)=-\sum_{k=1}^{P} a_{i}(k) c_{i}(n-k)+w_{i}(n)$

where $\boldsymbol{a}_{i}=\left[a_{i}(1), \ldots, a_{i}(P)\right]^{T}$ is the $P$-dimensional vector of complex forward AR coefficients in the range cell $i$ and $w_{i}(n)$ is zero mean complex Gaussian white noise with variance $\tau_{i}$ [22]. Under the AR model [16], we can rewrite (2) as:

$\left\{\begin{array}{c}\mathcal{H}_{0}: z_{i}(n)=-\sum_{k=1}^{P} a_{i}(k) z_{i}(n-k)+w_{i}(n) \\ i=1, \ldots, L ; n=P+1, \ldots, N \\ \mathcal{H}_{1}: z_{i}(n)=-\sum_{k=1}^{P} a_{i}(k)\left(z_{i}(n-k)-\alpha_{i} p(n-k)\right) \\ \quad+\alpha_{i} p(n)+w_{i}(n) \\ i=1, \ldots, L ; n=P+1, \ldots, N\end{array}\right.$

The conditional pdfs (3) and (4) can be approximated for $N \gg P$ [18,23], respectively, by

$f\left(\boldsymbol{z}_{i} \mid \mathcal{H}_{0} ; \tau_{i}, \boldsymbol{a}_{i}\right) \simeq \frac{1}{\left(\pi \tau_{i}\right)^{N-P}} \exp \left(-\frac{\left(\tilde{\boldsymbol{z}}_{i}+\widetilde{\boldsymbol{Z}}_{i} \boldsymbol{a}_{i}\right)^{H}\left(\tilde{\boldsymbol{z}}_{i}+\widetilde{\boldsymbol{Z}}_{i} \boldsymbol{a}_{i}\right)}{\tau_{i}}\right)$

$f\left(\boldsymbol{z}_{i} \mid \mathcal{H}_{1} ; \alpha_{i}, \tau_{i}, \boldsymbol{a}_{i}\right) \simeq \frac{1}{\left(\pi \tau_{i}\right)^{N-P}}$

$\times \exp \left(-\frac{\left(\tilde{\boldsymbol{z}}_{i}+\widetilde{\boldsymbol{Z}}_{i} \boldsymbol{a}_{i}-\alpha_{i}\left(\tilde{\boldsymbol{p}}+\tilde{\boldsymbol{P}} \boldsymbol{a}_{i}\right)\right)^{H}\left(\tilde{\boldsymbol{z}}_{i}+\widetilde{\boldsymbol{Z}}_{i} \boldsymbol{a}_{i}-\alpha_{i}\left(\tilde{\boldsymbol{p}}+\tilde{\boldsymbol{P}} \boldsymbol{a}_{i}\right)\right)}{\tau_{i}}\right)$ 
where the $(N-P)$ dimensional vectors $\tilde{\boldsymbol{z}}_{i}$ and $\tilde{\boldsymbol{p}}$ are given by $\tilde{\boldsymbol{z}}_{i}=$ $\left[z_{i}(P+1), \ldots, z_{i}(N)\right]^{T}$ and $\tilde{\boldsymbol{p}}=[p(P+1), \ldots, p(N)]^{T}$, whereas, the $(N-P) \times P$-dimensional matrices $\widetilde{\boldsymbol{Z}}_{i}$ and $\tilde{\boldsymbol{P}}$ are expressed by

$\widetilde{\boldsymbol{Z}}_{i}=\left[\begin{array}{cccc}z_{i}(P) & z_{i}(P-1) & \ldots & z_{i}(1) \\ z_{i}(P+1) & z_{i}(P) & \ldots & z_{i}(2) \\ \vdots & \vdots & \ddots & \vdots \\ z_{i}(N-1) & z_{i}(N-2) & \ldots & z_{i}(N-P)\end{array}\right]$

and

$\tilde{\boldsymbol{P}}=\left[\begin{array}{cccc}p(P) & p(P-1) & \ldots & p(1) \\ p(P+1) & p(P) & \ldots & p(2) \\ \vdots & \vdots & \ddots & \vdots \\ p(N-1) & p(N-2) & \ldots & p(N-P)\end{array}\right]$.

In the above equations, we used samples from $P+1$ to $N$ to model the received observation in a forward manner. In the following, we propose to extend this modeling by adding the prediction of samples from 1 to $P$, in a backward manner in order to gain more information, thus enhancing the performance of the proposed detector.

\subsection{The proposed forward-backward modeling}

In the backward prediction, we propose to predict the $(n-P)$ th sample from the $P$ future samples. Thus, for $n=P+1, \ldots, N$ and $i=1, \ldots, L$, we obtain

$c_{i}(n-P)=-\sum_{k=1}^{P} b_{i}(k) c_{i}(n-k+1)+w_{i}(n)$

where $\boldsymbol{b}_{i}=\left[b_{i}(1), \ldots, b_{i}(P)\right]^{T}$ is the $P$-dimensional vector of complex backward AR coefficients in the $i$-th range cell and $w_{i}(n)$ denotes a zero mean complex Gaussian white noise with variance $\tau_{i}[22]$.

In the backward context, we can rewrite (2), for $n=P+$ $1, \ldots, N$ and $i=1, \ldots, L$,

$\left\{\begin{array}{rr}\mathcal{H}_{0}: \quad z_{i}(n-P)=- & \sum_{k=1}^{P} b_{i}(k) z_{i}(n-k+1)+w_{i}(n) \\ \mathcal{H}_{1}: \quad z_{i}(n-P)= & -\sum_{k=1}^{P} b_{i}(k)\left(z_{i}(n-k+1)\right. \\ & \left.\quad-\alpha_{i} p(n-k+1)\right)+\alpha_{i} p(n-P)+w_{i}(n) .\end{array}\right.$

Let

$\boldsymbol{z}_{i}=\left[\breve{\boldsymbol{z}}_{i}^{T} \tilde{\boldsymbol{z}}_{i}^{T}\right]^{T}$

in which $\breve{\boldsymbol{z}}_{i}=\left[z_{i}(1), \ldots, z_{i}(P)\right]^{T}$ and $\tilde{\boldsymbol{z}}_{i}=\left[z_{i}(P+1), \ldots, z_{i}(N)\right]^{T}$. In the following, samples of the vector $\breve{z}_{i}$ are predicted by a backward manner and samples of the vector $\tilde{\boldsymbol{z}}_{i}$ are predicted by a forward manner.

\subsubsection{Prediction under $\mathcal{H}_{0}$ hypothesis}

Under $\mathcal{H}_{0}$ hypothesis and from (10), the backward prediction of $\breve{z}_{i}$ is given by

$\hat{\breve{z}}_{i}=-\breve{\boldsymbol{Z}}_{i} \boldsymbol{b}_{i}$

with

$\breve{\boldsymbol{Z}}_{i}=\left[\begin{array}{cccc}z_{i}(P+1) & z_{i}(P) & \ldots & z_{i}(2) \\ z_{i}(P+2) & z_{i}(P+1) & \ldots & z_{i}(3) \\ \vdots & \vdots & \ddots & \vdots \\ z_{i}(2 P) & z_{i}(2 P-1) & \ldots & z_{i}(P+1)\end{array}\right]$.
Using (6), the forward prediction of $\tilde{\boldsymbol{z}}_{i}$ is given by

$\hat{\tilde{\boldsymbol{z}}}_{i}=-\widetilde{\boldsymbol{Z}}_{i} \boldsymbol{a}_{i}$.

Consequently, from (12) and (14), and under $\mathcal{H}_{0}$, the forwardbackward prediction of $z_{i}$ is given by

$\hat{\boldsymbol{z}}_{i}=\left[\begin{array}{c}\hat{\boldsymbol{z}}_{i} \\ \hat{\tilde{\boldsymbol{z}}}_{i}\end{array}\right]=-\boldsymbol{Z}_{i} \boldsymbol{d}_{i}$

with $\boldsymbol{Z}_{i}=\operatorname{bdiag}\left(\breve{\boldsymbol{Z}}_{i}, \widetilde{\boldsymbol{Z}}_{i}\right)$ and $\boldsymbol{d}_{i}=\left[\boldsymbol{b}_{i}^{T}, \boldsymbol{a}_{i}^{T}\right]^{T}$. The residual of the forward-backward prediction of $\boldsymbol{z}_{i}$ is given by

$\boldsymbol{w}_{i}=\boldsymbol{z}_{i}-\hat{\boldsymbol{z}}_{i}=\boldsymbol{z}_{i}+\boldsymbol{Z}_{i} \boldsymbol{d}_{i}$

Then, the conditional pdf (3), under $\mathcal{H}_{0}$, can be approximated for $N \gg P$ by [23]

$f\left(\boldsymbol{z}_{i} \mid \mathcal{H}_{0} ; \tau_{i}, \boldsymbol{d}_{i}\right) \simeq \frac{1}{\left(\pi \tau_{i}\right)^{N}} \exp \left(-\frac{\left(\boldsymbol{z}_{i}+\boldsymbol{Z}_{i} \boldsymbol{d}_{i}\right)^{H}\left(\boldsymbol{z}_{i}+\boldsymbol{Z}_{i} \boldsymbol{d}_{i}\right)}{\tau_{i}}\right)$

\subsubsection{Prediction under $\mathcal{H}_{1}$ hypothesis}

Let us denote

$\mathbf{s}_{i}=\left[\breve{\boldsymbol{s}}_{i}^{T} \tilde{\boldsymbol{s}}_{i}^{T}\right]^{T}$

with

$\breve{\boldsymbol{s}}_{i}=\left[s_{i}(1), \ldots, s_{i}(P)\right]^{T}$,

$\tilde{\boldsymbol{s}}_{i}=\left[s_{i}(P+1), \ldots, s_{i}(N)\right]^{T}$

in which, $s_{i}(n)=z_{i}(n)-\alpha_{i} p(n)$, for $n=1, \ldots, N$. Under $\mathcal{H}_{1}$ hypothesis and from (10), the backward prediction of $\breve{\boldsymbol{s}}_{i}$ conditioned to $\alpha_{i}$, is given by

$\hat{\breve{s}}_{i}=-\breve{\boldsymbol{S}}_{i} \boldsymbol{b}_{i}$

with

$\breve{\boldsymbol{S}}_{i}=\left[\begin{array}{cccc}s_{i}(P+1) & s_{i}(P) & \ldots & s_{i}(2) \\ s_{i}(P+2) & s_{i}(P+1) & \ldots & s_{i}(3) \\ \vdots & \vdots & \ddots & \vdots \\ s_{i}(2 P) & s_{i}(2 P-1) & \ldots & s_{i}(P+1)\end{array}\right]$.

On the other hand, from (6), the forward prediction of $\tilde{\boldsymbol{s}}_{i}$ conditioned to $\alpha_{i}$, can be expressed by

$\hat{\tilde{\boldsymbol{s}}}_{i}=-\tilde{\boldsymbol{S}}_{i} \boldsymbol{a}_{i}$

with

$\tilde{\boldsymbol{S}}_{i}=\left[\begin{array}{cccc}s_{i}(P) & s_{i}(P-1) & \ldots & s_{i}(1) \\ s_{i}(P+1) & s_{i}(P) & \ldots & s_{i}(2) \\ \vdots & \vdots & \ddots & \vdots \\ s_{i}(N-1) & s_{i}(N-2) & \ldots & s_{i}(N-P)\end{array}\right]$.

Finally, from (21) and (23), the forward-backward prediction of $\mathbf{s}_{i}$ under $\mathcal{H}_{1}$ is given by

$\hat{\mathbf{s}}_{i}=\left[\begin{array}{l}\hat{\boldsymbol{s}}_{i} \\ \hat{\tilde{\boldsymbol{s}}}_{i}\end{array}\right]$

and its error vector is expressed as

$\boldsymbol{w}_{i}=\mathbf{s}_{i}-\hat{\mathbf{s}}_{i}=\left[\begin{array}{c}\breve{\boldsymbol{w}} \\ \tilde{\boldsymbol{w}}\end{array}\right]$ 
in which

$\breve{\boldsymbol{w}}=\breve{\boldsymbol{s}}_{i}+\breve{\boldsymbol{S}}_{i} \boldsymbol{b}_{i}$

and

$\tilde{\boldsymbol{w}}=\tilde{\boldsymbol{s}}_{i}+\tilde{\boldsymbol{S}}_{i} \boldsymbol{a}_{i}$.

In addition, using (10) and (19), we have

$\breve{\boldsymbol{s}}_{i}=\breve{\boldsymbol{z}}_{i}-\alpha_{i} \breve{\boldsymbol{p}}$,

$\breve{\boldsymbol{S}}_{i}=\breve{\boldsymbol{Z}}_{i}-\alpha_{i} \breve{\boldsymbol{P}}$

with $\breve{\boldsymbol{p}}=[p(1), \ldots, p(P)]^{T}$ and

$\breve{\boldsymbol{P}}=\left[\begin{array}{ccc}p(P+1) & \ldots & p(2) \\ \vdots & \ddots & \vdots \\ p(2 P) & \ldots & p(P+1)\end{array}\right]$.

Then, from (27), (29), (30) and after straightforward manipulations, we obtain

$\breve{\boldsymbol{w}}=\breve{\boldsymbol{z}}_{i}+\breve{\boldsymbol{Z}}_{i} \boldsymbol{b}_{i}-\alpha_{i}\left(\breve{\boldsymbol{p}}+\breve{\boldsymbol{P}} \boldsymbol{b}_{i}\right)$.

Using the same methodology for $\tilde{\boldsymbol{w}}$, we have from (6) and (20):

$\tilde{\boldsymbol{s}}_{i}=\tilde{\boldsymbol{z}}_{i}-\alpha_{i} \tilde{\boldsymbol{p}}$.

On the other hand, (6) and (24) lead to

$\tilde{\boldsymbol{S}}_{i}=\widetilde{\boldsymbol{Z}}_{i}-\alpha_{i} \tilde{\boldsymbol{P}}$

Consequently, combining (28), (33) and (34), we obtain

$\tilde{\boldsymbol{w}}=\tilde{\boldsymbol{z}}_{i}+\widetilde{\boldsymbol{Z}}_{i} \boldsymbol{a}_{i}-\alpha_{i}\left(\tilde{\boldsymbol{p}}+\tilde{\boldsymbol{P}} \boldsymbol{a}_{i}\right)$

Finally, from (32) and (35), the forward-backward residual vector of $\mathbf{s}_{i}$ conditioned to $\alpha_{i}$ is given by

$\boldsymbol{w}_{i}=\boldsymbol{z}_{i}+\boldsymbol{Z}_{i} \boldsymbol{d}_{i}-\alpha_{i}\left(\mathbf{p}+\boldsymbol{P} \boldsymbol{d}_{i}\right)$

with $\boldsymbol{P}=\operatorname{bdiag}(\breve{\boldsymbol{P}}, \tilde{\boldsymbol{P}})=\left[\begin{array}{cc}\breve{\boldsymbol{P}} & \mathbf{0} \\ \mathbf{0} & \tilde{\boldsymbol{P}}\end{array}\right]$.

In summary, using the same methodology as for $\mathcal{H}_{0}$, we conclude that the conditional pdf given in (4), under $\mathcal{H}_{1}$, can be approximated for $N \gg P$ by

$$
\begin{aligned}
& p\left(\boldsymbol{z}_{i} \mid \mathcal{H}_{1} ; \alpha_{i}, \tau_{i}, \boldsymbol{d}_{i}\right) \simeq \frac{1}{\left(\pi \tau_{i}\right)^{N}} \\
& \quad \times \exp \left(-\frac{\left(\boldsymbol{z}_{i}+\boldsymbol{Z}_{i} \boldsymbol{d}_{i}-\alpha_{i}\left(\mathbf{p}+\boldsymbol{P} \boldsymbol{d}_{i}\right)\right)^{H}\left(\boldsymbol{z}_{i}+\boldsymbol{Z}_{i} \boldsymbol{d}_{i}-\alpha_{i}\left(\mathbf{p}+\boldsymbol{P d _ { i } ) )}\right.\right.}{\tau_{i}}\right)
\end{aligned}
$$

\section{The proposed adaptive detection algorithm}

\subsection{Homogeneous environment}

In a homogeneous environment, it is commonly assumed that the clutter parameters do not change from cell to cell, which means that $\boldsymbol{d} \triangleq \boldsymbol{d}_{1}=\ldots=\boldsymbol{d}_{L}$, and $\tau \triangleq \tau_{1}=\ldots=\tau_{L}[17,18]$.

The optimum decision rule for target detection is the well known Neyman-Pearson criterion, which leads to the likelihood ratio test. Nevertheless, in our context, the parameters $\boldsymbol{\alpha}=$ $\left[\alpha_{1}, \ldots, \alpha_{L}\right], \tau$ and $\boldsymbol{d}$ are unknown. We therefore resort to the suboptimal GLRT detector given by

$\Lambda_{\mathrm{GLRT}}\left(\boldsymbol{z}_{1}, \ldots, \boldsymbol{z}_{L}\right)=\frac{\max _{\boldsymbol{\alpha}, \tau, \boldsymbol{d}} f\left(\boldsymbol{z}_{1}, \ldots, \boldsymbol{z}_{L} \mid \mathcal{H}_{1} ; \boldsymbol{\alpha}, \tau, \boldsymbol{d}\right)}{\max _{\tau, \boldsymbol{d}} f\left(\boldsymbol{z}_{1}, \ldots, \boldsymbol{z}_{L} \mid \mathcal{H}_{0} ; \tau, \boldsymbol{d}\right)} \underset{\mathcal{H}_{0}}{\stackrel{\mathcal{H}_{1}}{\gtrless} \delta}$ where $\delta$ denotes the detection threshold which is set according to the desired value of the false alarm probability [24].

Assuming independent $\boldsymbol{z}_{i}$ for $i=1, \ldots, L$ and using (17) and (37), the conditional joint pdfs of the total observation vectors $\boldsymbol{z}_{1}, \ldots, \boldsymbol{z}_{L}$ under $\mathcal{H}_{0}$ and $\mathcal{H}_{1}$ are, respectively, approximated by

$$
\begin{aligned}
& f\left(\boldsymbol{z}_{1}, \ldots, \boldsymbol{z}_{L} \mid \mathcal{H}_{0} ; \tau, \boldsymbol{d}\right) \\
& \quad \simeq \frac{1}{(\pi \tau)^{N L}} \exp \left(-\sum_{i=1}^{L} \frac{\left(\boldsymbol{z}_{i}+\boldsymbol{Z}_{i} \boldsymbol{d}\right)^{H}\left(\boldsymbol{z}_{i}+\boldsymbol{Z}_{i} \boldsymbol{d}\right)}{\tau}\right)
\end{aligned}
$$

and

$$
\begin{aligned}
& f\left(\boldsymbol{z}_{1}, \ldots, \boldsymbol{z}_{L} \mid \mathcal{H}_{1} ; \boldsymbol{\alpha}, \tau, \boldsymbol{d}\right) \simeq \frac{1}{(\pi \tau)^{N L}} \\
& \quad \times \exp \left(-\sum_{i=1}^{L} \frac{\left(\boldsymbol{z}_{i}+\boldsymbol{Z}_{i} \boldsymbol{d}-\alpha_{i}(\mathbf{p}+\boldsymbol{P d})\right)^{H}\left(\boldsymbol{z}_{i}+\boldsymbol{Z}_{i} \boldsymbol{d}-\alpha_{i}(\mathbf{p}+\boldsymbol{P d})\right)}{\tau}\right)
\end{aligned}
$$

in which, the estimation of the unknown parameters are performed by the maximum likelihood method in the following.

\subsubsection{Maximum likelihood estimate of $\boldsymbol{\alpha}$}

Under $\mathcal{H}_{1}$ hypothesis, we can rewrite (40) as:

$f\left(\boldsymbol{z}_{1}, \ldots, \boldsymbol{z}_{L} \mid \mathcal{H}_{1} ; \boldsymbol{\alpha}, \tau, \boldsymbol{d}\right) \simeq \frac{1}{(\pi \tau)^{N L}} \exp \left(-\sum_{i=1}^{L} \frac{\left|\mathbf{g}_{i}-\alpha_{i} \boldsymbol{\psi}\right|^{2}}{\tau}\right)$

in which $\mathbf{g}_{i}=\boldsymbol{z}_{i}+\boldsymbol{Z}_{i} \boldsymbol{d}$ and $\boldsymbol{\psi}=\mathbf{p}+\boldsymbol{P} \boldsymbol{d}$. The maximum likelihood estimator (MLE) of $\alpha_{i}$ is given by

$\hat{\alpha}_{i}=\arg \min _{\alpha_{i}}\left|\mathbf{g}_{i}-\alpha_{i} \boldsymbol{\psi}\right|^{2}=\frac{\boldsymbol{\psi}^{H} \mathbf{g}_{i}}{\boldsymbol{\psi}^{H} \boldsymbol{\psi}}$.

Plugging (42) into (41), we obtain

$$
\begin{aligned}
& f\left(\boldsymbol{z}_{1}, \ldots, \boldsymbol{z}_{L} \mid \mathcal{H}_{1} ; \hat{\boldsymbol{\alpha}}, \tau, \boldsymbol{d}\right) \\
& \quad \simeq \frac{1}{(\pi \tau)^{N L}} \exp \left(-\sum_{i=1}^{L} \frac{\left(\boldsymbol{z}_{i}+\boldsymbol{Z}_{i} \boldsymbol{d}\right)^{H} \boldsymbol{P}_{\psi}^{\perp}\left(\boldsymbol{z}_{i}+\boldsymbol{Z}_{i} \boldsymbol{d}\right)}{\tau}\right)
\end{aligned}
$$

where the orthogonal projector onto the subspace spanned by $\psi$ is given by $\boldsymbol{P}_{\psi}^{\perp}=\mathbf{I}_{N}-\frac{\psi \psi^{H}}{\psi^{H} \psi}$ in which $\mathbf{I}_{N}$ is the $N \times N$ identity matrix and $\hat{\boldsymbol{\alpha}}=\left[\hat{\alpha}_{1}, \ldots, \hat{\alpha}_{L}\right]^{T}$.

\subsubsection{Maximum likelihood estimates of $\tau$ and $\boldsymbol{d}$}

To find the maximum likelihood estimate of $\tau$ under $\mathcal{H}_{0}$ and $\mathcal{H}_{1}$, we maximize (39) and (43) with respect to $\tau$, respectively. After some manipulations, we obtain

$\hat{\tau}_{\mathcal{H}_{0}}=\frac{\sum_{i=1}^{L}\left(\boldsymbol{z}_{i}+\boldsymbol{Z}_{i} \boldsymbol{d}_{\mathcal{H}_{0}}\right)^{H}\left(\boldsymbol{z}_{i}+\boldsymbol{Z}_{i} \boldsymbol{d}_{\mathcal{H}_{0}}\right)}{N L}$

$\hat{\tau}_{\mathcal{H}_{1}}=\frac{\sum_{i=1}^{L}\left(\boldsymbol{z}_{i}+\boldsymbol{Z}_{i} \boldsymbol{d}_{\mathcal{H}_{1}}\right)^{H} \boldsymbol{P}_{\psi}^{\perp}\left(\boldsymbol{z}_{i}+\boldsymbol{Z}_{i} \boldsymbol{d}_{\mathcal{H}_{1}}\right)}{N L}$

Following the same methodology as in [18], the MLE of the forward part, i.e., $\boldsymbol{a}$ under $\mathcal{H}_{0}$ and $\mathcal{H}_{1}$, respectively, is given by

$\hat{\boldsymbol{a}}_{\mathcal{H}_{0}}=-\left(\sum_{i=1}^{L} \widetilde{\boldsymbol{Z}}_{i}^{H} \widetilde{\boldsymbol{Z}}_{i}\right)^{-1}\left(\sum_{i=1}^{L} \widetilde{\boldsymbol{Z}}_{i}^{H} \tilde{\boldsymbol{z}}_{i}\right)$

$\hat{\boldsymbol{a}}_{\mathcal{H}_{1}}=-\left(\sum_{i=1}^{L} \widetilde{\boldsymbol{Z}}_{i}^{H} \boldsymbol{P}_{\tilde{\boldsymbol{\phi}}}^{\perp} \widetilde{\mathbf{Z}}_{i}\right)^{-1}\left(\sum_{i=1}^{L} \widetilde{\boldsymbol{Z}}_{i}^{H} \boldsymbol{P}_{\tilde{\boldsymbol{\phi}}}^{\perp} \tilde{\boldsymbol{z}}_{i}\right)$ 
where the orthogonal projector onto the subspace spanned by $\tilde{\boldsymbol{\phi}}=\left[1, \ldots, e^{j 2 \pi f_{d}(N-P-1)}\right]^{T}$ is given by $\boldsymbol{P}_{\tilde{\boldsymbol{\phi}}}^{\perp}=\mathbf{I}_{N-P}-\frac{\tilde{\boldsymbol{\phi}} \tilde{\boldsymbol{\phi}}^{H}}{\tilde{\boldsymbol{\phi}} \tilde{\boldsymbol{\phi}}}$. Consequently, the backward coefficients are deduced, under $\mathcal{H}_{0}$ and $\mathcal{H}_{1}$, thanks to the following relationship (c.f. [22] for proof)

$b(k)=a^{*}(P-k+1), \quad k=1, \ldots, P$

4.1.3. Decision rule of the proposed forward-backward autoregressive GLRT detector

We replace the unknown parameters by their MLE estimates, in the joint conditional pdfs of the total observation $\boldsymbol{z}_{1}, \ldots, \boldsymbol{z}_{L}$ under $\mathcal{H}_{0}$ and $\mathcal{H}_{1}$, from (39) and (40), we find, respectively,

$$
\begin{aligned}
f & \left(\boldsymbol{z}_{1}, \ldots, \boldsymbol{z}_{L} \mid \mathcal{H}_{0} ; \hat{\tau}_{\mathcal{H}_{0}}, \hat{\boldsymbol{d}}_{\mathcal{H}_{0}}\right) \\
& \simeq\left[\frac{N L}{\pi \exp (1) \sum_{i=1}^{L}\left(\boldsymbol{z}_{i}+\boldsymbol{Z}_{i} \hat{\boldsymbol{d}}_{\mathcal{H}_{0}}\right)^{H}\left(\boldsymbol{z}_{i}+\boldsymbol{Z}_{i} \hat{\boldsymbol{d}}_{\mathcal{H}_{0}}\right)}\right]^{N L}
\end{aligned}
$$

and

$$
\begin{aligned}
f\left(\boldsymbol{z}_{1}, \ldots, \boldsymbol{z}_{L} \mid \mathcal{H}_{1} ; \hat{\boldsymbol{\alpha}}, \hat{\tau}_{\mathcal{H}_{1}}, \hat{\boldsymbol{d}}_{\mathcal{H}_{1}}\right) \\
\quad \simeq\left[\frac{N L}{\pi \exp (1) \sum_{i=1}^{L}\left(\boldsymbol{z}_{i}+\boldsymbol{Z}_{i} \hat{\boldsymbol{d}}_{\mathcal{H}_{1}}\right)^{H} \boldsymbol{P}_{\psi}^{\perp}\left(\boldsymbol{z}_{i}+\boldsymbol{Z}_{i} \hat{\boldsymbol{d}}_{\mathcal{H}_{1}}\right)}\right]^{N L} .
\end{aligned}
$$

where $\boldsymbol{P}_{\psi}^{\perp}$ is evaluated for $\hat{\boldsymbol{d}}_{\mathcal{H}_{1}}$.

Consequently, from the above equations, the decision rule of the proposed detector is given thanks to approximations (49) and (50), i.e.,

$\Lambda_{\mathrm{FBD}-\mathrm{H}}\left(\boldsymbol{z}_{1}, \ldots, \boldsymbol{z}_{L}\right)=\frac{\sum_{i=1}^{L}\left(\boldsymbol{z}_{i}+\boldsymbol{Z}_{i} \hat{\boldsymbol{d}}_{\mathcal{H}_{0}}\right)^{H}\left(\boldsymbol{z}_{i}+\boldsymbol{Z}_{i} \hat{\boldsymbol{d}}_{\mathcal{H}_{0}}\right)}{\sum_{i=1}^{L}\left(\boldsymbol{z}_{i}+\boldsymbol{Z}_{i} \hat{\boldsymbol{d}}_{\mathcal{H}_{1}}\right)^{H} \boldsymbol{P} \stackrel{\perp}{\perp}\left(\boldsymbol{z}_{i}+\boldsymbol{Z}_{i} \hat{\boldsymbol{d}}_{\mathcal{H}_{1}}\right)} \underset{\mathcal{H}_{0}}{\stackrel{\mathcal{H}_{1}}{\gtrless}} \delta$.

The proposed detector (51) is named forward-backward autoregressive GLRT detector in a homogeneous context (FBD-H). It uses all available samples (i.e., from 1 to $N$ ) in contrast to the ARGLRTCG detector given in [17] and the ARGLRT detector given in [18], which use only $N-P$ samples.

\subsection{Heterogeneous environment}

In a heterogeneous environment, the clutter parameters change in space. This means that the autoregressive coefficients $\boldsymbol{d}_{i}$, as well the clutter power $\tau_{i}$, change from range cell to range cell. As in the homogeneous environment context, we resort to the GLRT strategy in order to setup our detector. Let us denote $\boldsymbol{\alpha}=\left[\alpha_{1}, \ldots, \alpha_{L}\right]^{T}$, $\boldsymbol{\tau}=\left[\tau_{1}, \ldots, \tau_{L}\right]^{T}$ and $\boldsymbol{d}=\left[\boldsymbol{d}_{1}^{T}, \ldots, \boldsymbol{d}_{L}^{T}\right]^{T}$, which represent the unknown parameters. The GLRT is expressed as follows:

$\Lambda_{\mathrm{GLRT}}\left(\boldsymbol{z}_{1}, \ldots, \boldsymbol{z}_{L}\right)=\frac{\max _{\boldsymbol{\alpha}, \boldsymbol{\tau}, \boldsymbol{d}} f\left(\boldsymbol{z}_{1}, \ldots, \boldsymbol{z}_{L} \mid \mathcal{H}_{1} ; \boldsymbol{\alpha}, \boldsymbol{\tau}, \boldsymbol{a}\right)}{\max _{\boldsymbol{\tau}, \boldsymbol{d}} f\left(\boldsymbol{z}_{1}, \ldots, \boldsymbol{z}_{L} \mid \mathcal{H}_{0} ; \boldsymbol{\tau}, \boldsymbol{a}\right)} \underset{\mathcal{H}_{0}}{\stackrel{\mathcal{H}_{1}}{\gtrless} \delta .}$

Using (17) and (37), the conditional joint pdfs of the total observation under $\mathcal{H}_{0}$ and $\mathcal{H}_{1}$ and for independent $\boldsymbol{z}_{i}, i=1, \ldots, L$, are approximated, respectively, by

$$
\begin{aligned}
& f\left(\boldsymbol{z}_{1}, \ldots, \boldsymbol{z}_{L} \mid \mathcal{H}_{0} ; \boldsymbol{\tau}, \boldsymbol{d}\right) \\
& \quad \simeq \frac{1}{\prod_{i=1}^{L}\left(\pi \tau_{i}\right)^{N}} \exp \left(-\sum_{i=1}^{L} \frac{\left(\boldsymbol{z}_{i}+\boldsymbol{Z}_{i} \boldsymbol{d}_{i}\right)^{H}\left(\boldsymbol{z}_{i}+\boldsymbol{Z}_{i} \boldsymbol{d}_{i}\right)}{\tau_{i}}\right)
\end{aligned}
$$

$$
\begin{aligned}
& f\left(\boldsymbol{z}_{1}, \ldots, \boldsymbol{z}_{L} \mid \mathcal{H}_{1} ; \boldsymbol{\alpha}, \boldsymbol{\tau}, \boldsymbol{d}\right) \simeq \frac{1}{\prod_{i=1}^{L}\left(\pi \tau_{i}\right)^{N}} \\
& \quad \times \exp \left(-\sum_{i=1}^{L} \frac{\left(\boldsymbol{z}_{i}+\boldsymbol{Z}_{i} \mathbf{d}_{i}-\alpha_{i}\left(\mathbf{p}+\boldsymbol{P} \boldsymbol{d}_{i}\right)\right)^{H}\left(\boldsymbol{z}_{i}+\boldsymbol{Z}_{i} \boldsymbol{d}_{i}-\alpha_{i}\left(\mathbf{p}+\boldsymbol{P} \boldsymbol{d}_{i}\right)\right)}{\tau_{i}}\right)
\end{aligned}
$$

in which, the estimation of the unknown parameters will be performed by the maximum likelihood method in the following.

\subsubsection{Maximum likelihood estimate of $\boldsymbol{\alpha}$}

Let us denote, under $\mathcal{H}_{1}$ hypothesis, $\mathbf{g}_{i}=\boldsymbol{z}_{i}+\boldsymbol{Z}_{i} \boldsymbol{d}_{i}$ and $\boldsymbol{\psi}_{i}=$ $\mathbf{p}+\boldsymbol{P} \boldsymbol{d}_{i}$. Then (54) can be rewritten as

$$
\begin{aligned}
& f\left(\boldsymbol{z}_{1}, \ldots, \boldsymbol{z}_{L} \mid \mathcal{H}_{1} ; \boldsymbol{\alpha}, \boldsymbol{\tau}, \boldsymbol{d}\right) \\
& \quad \simeq \frac{1}{\prod_{i=1}^{L}\left(\pi \tau_{i}\right)^{N}} \exp \left(-\sum_{i=1}^{L} \frac{\left|\mathbf{g}_{i}-\alpha_{i} \boldsymbol{\psi}_{i}\right|^{2}}{\tau_{i}}\right) .
\end{aligned}
$$

The MLE of $\alpha_{i}$ is given by

$\hat{\alpha}_{i}=\frac{\boldsymbol{\psi}_{i}{ }^{H} \mathbf{g}_{i}}{\boldsymbol{\psi}_{i}{ }^{H} \boldsymbol{\psi}_{i}}$.

Plugging (56) into (55), we obtain

$\left|\mathbf{g}_{i}-\hat{\alpha}_{i} \boldsymbol{\psi}_{i}\right|^{2}=\left|\mathbf{g}_{i}\right|^{2}-\frac{\left|\mathbf{g}_{i}{ }^{H} \boldsymbol{\psi}_{i}\right|^{2}}{\boldsymbol{\psi}_{i}{ }^{H} \boldsymbol{\psi}_{i}}=\mathbf{g}_{i}{ }^{H} \boldsymbol{P}_{\boldsymbol{\psi}_{i}}^{\perp} \mathbf{g}_{i}$

in which the orthogonal projector onto the subspace $\psi$ is given by

$\boldsymbol{P}_{\psi_{i}}^{\perp}=\mathbf{I}_{N}-\frac{\boldsymbol{\psi}_{i} \boldsymbol{\psi}_{i}{ }^{H}}{\boldsymbol{\psi}_{i}{ }^{H} \boldsymbol{\psi}_{i}}$,

leading to

$$
\begin{aligned}
& f\left(\boldsymbol{z}_{1}, \ldots, \boldsymbol{z}_{L} \mid \mathcal{H}_{1} ; \hat{\boldsymbol{\alpha}}, \boldsymbol{\tau}, \boldsymbol{d}\right) \simeq \frac{1}{\prod_{i=1}^{L}\left(\pi \tau_{i}\right)^{N}} \\
& \quad \times \exp \left(-\sum_{i=1}^{L} \frac{\left(\boldsymbol{z}_{i}+\boldsymbol{Z}_{i} \boldsymbol{d}_{i}\right)^{H} \boldsymbol{P}_{\psi_{i}}^{\perp}\left(\boldsymbol{z}_{i}+\boldsymbol{Z}_{i} \boldsymbol{d}_{i}\right)}{\tau_{i}}\right) .
\end{aligned}
$$

\subsubsection{Maximum likelihood estimates of $\boldsymbol{\tau}$ and $\boldsymbol{d}$}

To find the maximum likelihood estimate of $\tau_{i}$ under $\mathcal{H}_{0}$ and $\mathcal{H}_{1}$ we maximize (53) and (59) with respect to $\tau_{i}$, respectively. After some manipulations, we obtain

$$
\begin{aligned}
\hat{\tau}_{\mathcal{H}_{0}, i} & =\frac{\left(\boldsymbol{z}_{i}+\boldsymbol{Z}_{i} \hat{\boldsymbol{d}}_{\mathcal{H}_{0}, i}\right)^{H}\left(\boldsymbol{z}_{i}+\boldsymbol{Z}_{i} \hat{\boldsymbol{d}}_{\mathcal{H}_{0}, i}\right)}{N} \\
\hat{\tau}_{\mathcal{H}_{1}, i} & =\frac{\left(\boldsymbol{z}_{i}+\boldsymbol{Z}_{i} \hat{\boldsymbol{d}}_{\mathcal{H}_{1}, i}\right)^{H} \boldsymbol{P}_{\psi_{i}}^{\perp}\left(\boldsymbol{z}_{i}+\boldsymbol{Z}_{i} \hat{\boldsymbol{d}}_{\mathcal{H}_{1}, i}\right)}{N}
\end{aligned}
$$

Following the same methodology as in [18], the MLE of the forward coefficients under $\mathcal{H}_{0}$ and $\mathcal{H}_{1}$, are given, respectively, by

$\hat{\boldsymbol{a}}_{\mathcal{H}_{0}, i}=-\left(\widetilde{\boldsymbol{Z}}_{i}^{H} \widetilde{\boldsymbol{Z}}_{i}\right)^{-1}\left(\widetilde{\boldsymbol{Z}}_{i}^{H} \tilde{\boldsymbol{z}}_{i}\right)$

and

$\hat{\boldsymbol{a}}_{\mathcal{H}_{1}, i}=-\left(\widetilde{\boldsymbol{Z}}_{i}^{H} \boldsymbol{P}_{\tilde{\boldsymbol{\phi}}}^{\perp} \widetilde{\boldsymbol{Z}}_{i}\right)^{-1}\left(\widetilde{\boldsymbol{Z}}_{i}^{H} \boldsymbol{P}_{\tilde{\boldsymbol{\phi}}}^{\perp} \tilde{\boldsymbol{z}}_{i}\right)$.

Then, we deduce the backward coefficients estimation thanks to $b_{i}(k)=a_{i}^{*}(P-k+1), k=1, \ldots, P[22]$. 
4.2.3. Decision rule of the proposed forward-backward autoregressive GLRT detector

Let us replace the unknown parameters by their MLE estimates, in the joint conditional pdfs of the total observation vector under $\mathcal{H}_{0}$ and $\mathcal{H}_{1}$, from (53) and (54), we find, respectively,

$$
\begin{aligned}
& f\left(\boldsymbol{z}_{1}, \ldots, \boldsymbol{z}_{L} \mid \mathcal{H}_{0} ; \hat{\boldsymbol{\tau}}_{\mathcal{H}_{0}}, \hat{\boldsymbol{d}}_{\mathcal{H}_{0}}\right) \\
& \quad \simeq\left[\frac{N^{L}}{\pi^{L} \exp (L) \prod_{i=1}^{L}\left(\boldsymbol{z}_{i}+\boldsymbol{Z}_{i} \hat{\boldsymbol{d}}_{\mathcal{H}_{0}, i}\right)^{H}\left(\boldsymbol{z}_{i}+\boldsymbol{Z}_{i} \hat{\boldsymbol{d}}_{\mathcal{H}_{0}, i}\right)}\right]^{N}
\end{aligned}
$$

and

$$
\begin{aligned}
f & \left(\boldsymbol{z}_{1}, \ldots, \boldsymbol{z}_{L} \mid \mathcal{H}_{1} ; \hat{\boldsymbol{\alpha}}, \hat{\boldsymbol{\tau}}_{\mathcal{H}_{1}}, \hat{\boldsymbol{d}}_{\mathcal{H}_{1}}\right) \\
& \simeq\left[\frac{N^{L}}{\pi^{L} \exp (L) \prod_{i=1}^{L}\left(\boldsymbol{z}_{i}+\boldsymbol{Z}_{i} \hat{\boldsymbol{d}}_{\mathcal{H}_{1}, i}\right)^{H} \boldsymbol{P}_{\psi_{i}}^{\perp}\left(\boldsymbol{z}_{i}+\boldsymbol{Z}_{i} \hat{\boldsymbol{d}}_{\mathcal{H}_{1}, i}\right)}\right]^{N}
\end{aligned}
$$

Consequently, from the above equations, we obtain the following decision rule

$\Lambda_{\mathrm{FBD}-\mathrm{T}}\left(\boldsymbol{z}_{1}, \ldots, \boldsymbol{z}_{L}\right)=\prod_{i=1}^{L} \frac{\left(\boldsymbol{z}_{i}+\boldsymbol{Z}_{i} \hat{\boldsymbol{d}}_{\mathcal{H}_{0}, i}\right)^{H}\left(\boldsymbol{z}_{i}+\boldsymbol{Z}_{i} \hat{\boldsymbol{d}}_{\mathcal{H}_{0}, i}\right)}{\left(\boldsymbol{z}_{i}+\boldsymbol{Z}_{i} \hat{\boldsymbol{d}}_{\mathcal{H}_{1}, i}\right)^{H} \boldsymbol{P}_{\psi_{i}}^{\perp}\left(\boldsymbol{z}_{i}+\boldsymbol{Z}_{i} \hat{\boldsymbol{d}}_{\mathcal{H}_{1}, i}\right)} \underset{\mathcal{H}_{0}}{\stackrel{\mathcal{H}_{1}}{\gtrless}} \delta$

in which $\boldsymbol{P} \underset{\psi_{i}}{\perp}$ is evaluated for $\hat{\boldsymbol{d}}_{\mathcal{H}_{1}, i}$.

We name this proposed detector as forward-backward autoregressive GLRT detector in a heterogeneous environment (FBD-T). Unlike ARGLRT-HTG [17] and [18], FBD-T uses all available samples, i.e., from 1 to $N$. We will demonstrate in the next section that the proposed detectors, namely, FBD-H and FBD-T, have better performance compared respectively to the ARGLRT and ARGLRTHTG detectors for small data records, both in homogeneous and heterogeneous environment.

\section{Performance analysis}

This section is dedicated to numerical analysis of the proposed detectors in the homogeneous and heterogeneous contexts.

In both contexts, we perform a numerical comparison between the detectors ARGLRT, ARGLRT-HTG [18], the AML-GLRT $[13,19]$ and the proposed FBD-H and FBD-T detectors, in terms of the false alarm probability $P_{\mathrm{fa}}$ and the detection probability $P_{\mathrm{d}}$.

\subsection{Simulation setup}

The standard Monte Carlo technique based on $100 / P_{\mathrm{fa} 0}$ and 10000 independent trials is used to estimate $P_{\mathrm{fa}}$ and $P_{\mathrm{d}}$ respectively, for a nominal value of the false alarm probability $P_{\mathrm{fa} 0}=$ $10^{-4}$ and a number of cells under test $L=8$. Furthermore, the order of the AR model is set to $P=3$, which is expected to give good performance for radar sea clutter $[17,18]$.

The covariance matrix is derived according to an exponentcorrelation structure [17] given by

$$
[\mathbf{M}]_{m, n}=\rho^{|m-n|}, 1 \leq m, n \leq N, 0 \leq \rho \leq 1
$$

where $\rho$ represents the one-lag correlation coefficient. Typical values of $\rho$ for radar sea clutter are in the interval $[0.9,0.99]$. For these simulations, we set, e.g., $\rho=0.92$ [19].

In the following, the clutter is assumed to be K-distributed, which models well the sea clutter [25]. In this latter case, the texture is gamma distributed as
$f_{\tau}\left(\tau_{i}\right)=\frac{1}{\Gamma(\nu)}\left(\frac{v}{\mu}\right)^{\nu} \tau_{i}^{\nu-1} \exp \left(-\frac{v}{\mu} \tau_{i}\right) u\left(\tau_{i}\right) \quad i=1, \ldots, L$

where $\Gamma$ (.) denotes the gamma function, $\mu$ and $v$ are, respectively, the scale and shape parameters and $u($.$) is the standard unit step$ function. Typical values of $v$ for sea clutter are greater than 0.4 . Let us consider $v=0.5$, which represents a spiky clutter situation [12].

\subsection{Background on the AML-GLRT detector}

In the following, we give a brief description of the GLRT detector using the so-called AML estimator. We assume that the receiver collects $K$ i.i.d. vectors of secondary data $\mathbf{z}_{k}=\left\{\sqrt{\tau_{k}} \mathbf{x}_{k}\right\}_{k=1}^{K}$ from $K$ adjacent range cells. The AML estimator of the covariance matrix is given for $K$ i.i.d. vectors of secondary data as

$\hat{\mathbf{M}}_{\mathrm{AML}}(m+1)=\frac{N}{K} \sum_{k=1}^{K} \frac{\mathbf{z}_{k} \mathbf{z}_{k}^{H}}{\mathbf{z}_{k}^{H} \hat{\mathbf{M}}_{\mathrm{AML}}^{-1}(m) \mathbf{z}_{k}}$

with $m=1, \ldots, t$ and $t$ denotes the iteration number [19]. Regarding the initialization, we use the normalized sample covariance matrix estimator, i.e.,

$\hat{\mathbf{M}}_{\mathrm{AML}}(1)=\hat{\mathbf{M}}_{\mathrm{NSCM}}=\frac{N}{K} \sum_{k=1}^{K} \frac{\mathbf{z}_{k} \mathbf{z}_{k}^{H}}{\mathbf{z}_{k}^{H} \mathbf{z}_{k}}$.

The convergence criterion used for the iterative algorithm is given by [13]

$C(m)=\frac{\left\|\hat{\mathbf{M}}_{\mathrm{AML}}(m+1)-\hat{\mathbf{M}}_{\mathrm{AML}}(m)\right\|}{\left\|\hat{\mathbf{M}}_{\mathrm{AML}}(m)\right\|} \leq 10^{-3}$

where $\|$.$\| denotes the Frobenius norm.$

In our scenario, the number of iterations $t$ is found to be equal to 3 most of the time. Greater values do not lead to further performance improvements, this result was also noticed in [13] and [19].

Finally, the AML-GLRT detector based on the AML estimator is given by [26]

$$
\begin{aligned}
& \Lambda_{\mathrm{AML}-\mathrm{GLRT}}\left(\boldsymbol{z}_{1}, \ldots, \boldsymbol{z}_{L}, \mathbf{z}_{1}, \ldots, \mathbf{z}_{K}\right) \\
& \quad=-N \sum_{i=1}^{L} \ln \left(1-\frac{\left|\mathbf{p}^{H} \hat{\mathbf{M}}_{\mathrm{AML}}^{-1} \boldsymbol{z}_{i}\right|^{2}}{\left(\boldsymbol{z}_{i}^{H} \hat{\mathbf{M}}_{\mathrm{AML}}^{-1} \boldsymbol{z}_{i}\right)\left(\mathbf{p}^{H} \hat{\mathbf{M}}_{\mathrm{AML}}^{-1} \mathbf{p}\right)}\right) \underset{\mathcal{H}_{0}}{\stackrel{\mathcal{H}_{1}}{\gtrless}} \delta
\end{aligned}
$$

\subsection{Performance in terms of false alarm probability}

- From Fig. 1, we note that the FBD-H detector and the AMLGLRT detector outperforms the ARGLRT detector in terms of the CFAR property, especially for $f_{\mathrm{d}}=0$, where we have a maximum in the power spectral density (PSD) of the clutter. This is due to the use of the whole sample set in the prediction procedure of the FBD-H detector, while the ARGLRT scheme uses only $N-P$ samples.

On the other hand, we observe comparable performance of the AML-GLRT and the proposed FBD-H detector. Nevertheless, it is worth mentioning that the AML-GLRT uses a large number of secondary data (in this case $K=64$ ), which are supposed to contain clutter only, to estimate the clutter covariance matrix, whereas, the FBD-H uses only $L=8$ cells under test without the strong assumptions of homogeneous and not corrupted secondary data.

- In Fig. 2, we compare the false alarm probabilities of the FBD$\mathrm{H}$, ARGLRT and AML-GLRT detectors for different values of $N$ in a homogeneous context. We observe that the proposed FBD$\mathrm{H}$ detector and the AML-GLRT detector have almost a constant 


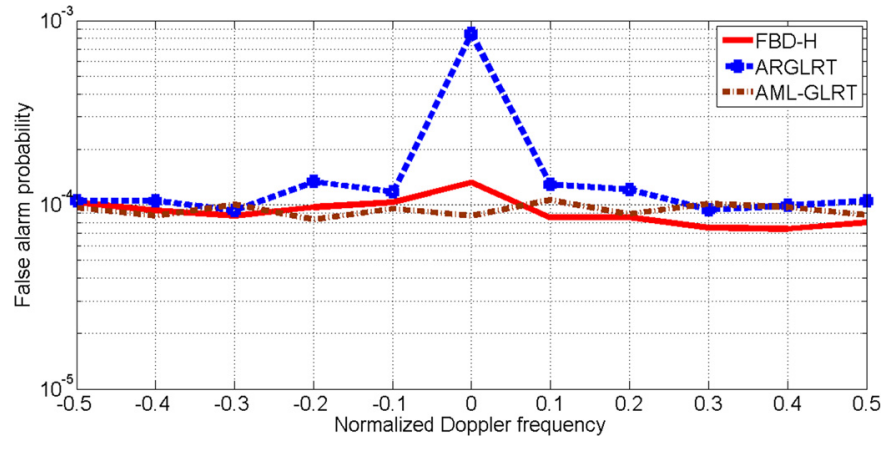

Fig. 1. $P_{\mathrm{fa}}$ versus $f_{\mathrm{d}}$ for $N=32$ and $K=64$ in a homogeneous context.

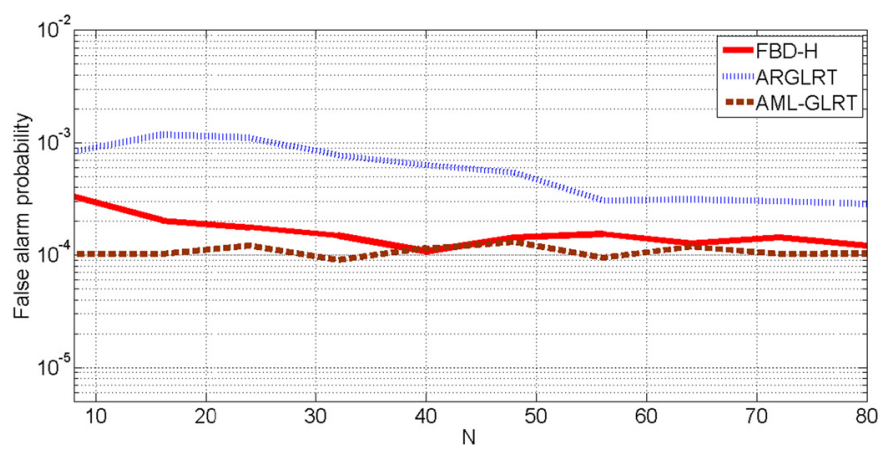

Fig. 2. $P_{\mathrm{fa}}$ versus $N$ for $f_{\mathrm{d}}=0$.

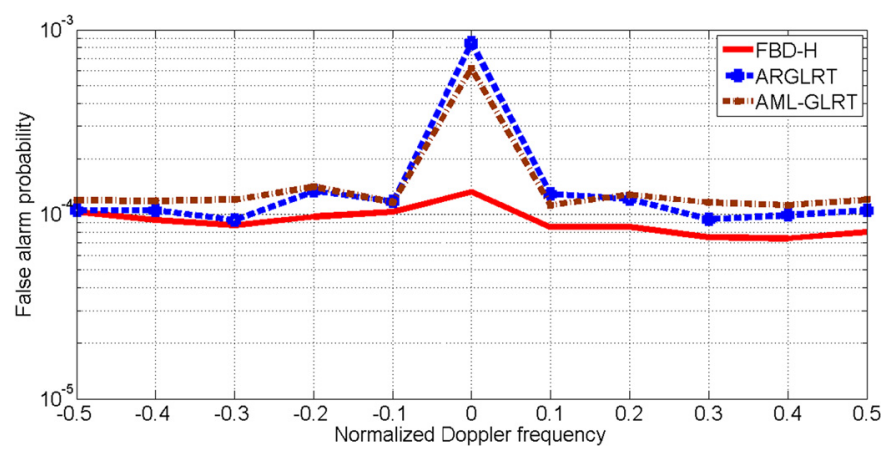

Fig. 3. $P_{\mathrm{fa}}$ versus $f_{\mathrm{d}}$ for $N=32, K=64$ in a non-homogeneous secondary data context.

false alarm probability equal to the nominal value $P_{\mathrm{fa} 0}$, contrary to the classical detector ARGLRT which has higher value of the $P_{\mathrm{fa}}$.

- Let us focus on the robustness of the aforementioned detectors w.r.t. non-homogeneities of the secondary data. We assume that the primary and the secondary data do not share the same covariance matrix. Specifically, let us assume a slight difference of the correlation coefficient $\rho_{\mathrm{p}}=0.92$ for the primary data, whereas, the first half of the secondary data share a correlation coefficient $\rho_{\mathrm{s}, 1}=0.9$ and the other half of the secondary data share a correlation coefficient $\rho_{\mathrm{s}, 2}=0.91$. The probability of false alarm for such scenario is represented in Fig. 3. From this figure, we notice, as expected, a severe degradation of the false alarm probability of the AML-GLRT detector compared with the FBD-H detector. This represents one of the major drawbacks of the AML-GLRT detector whereas the proposed detector remains robust in such scenario.

- In order to consider the heterogeneous version FBD-T of the proposed detector and the heterogeneous version ARGLRT-HTG of the classical detector, we assume a K-distributed clutter

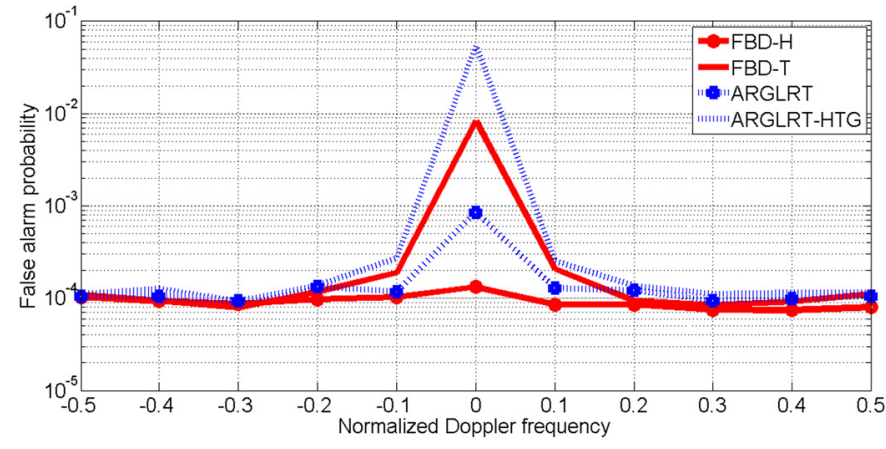

Fig. 4. $P_{\mathrm{fa}}$ versus $f_{\mathrm{d}}$ for $N=32, K=64$ of FBD-H, FBD-T, ARGLRT and ARGLRT-HTG detectors.

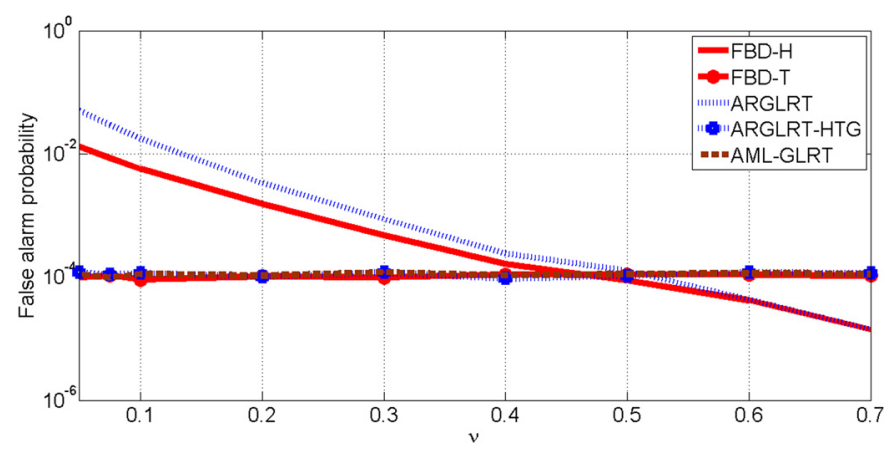

Fig. 5. $P_{\mathrm{fa}}$ versus $v$ for $N=8$.

data described in the beginning of section 5 . The parameters are set as $\rho=0.92, v=0.5, \mu=1$ and $L=8$.

In Fig. 4, we plot the false alarm probability vs. the normalized Doppler frequency of the detectors FBD-H, FBD-T, ARGLRT and ARGLRT-HTG. As expect, the homogeneous version of the proposed algorithm, namely FBD-H, outperforms the ARGLRT detector and its heterogeneous version ARGLRT-HTG. This is due to the spikiness nature of the clutter, for which the estimated value of the clutter power for each range cell is injected on the detector, knowing that, the clutter power changes from range cell to range cell.

- In Fig. 5, we perform a numerical analysis of the false alarm probability w.r.t. different values of the shape parameter $v$ for $N=8$. We observe that the ARGLRT-HTG, FBD-T and AMLGLRT detectors have the CFAR property with respect to $v$. This is due to the fact that the aforementioned detectors take into account the heterogeneous nature of the clutter and perform a normalization with respect to the clutter power in each range cell.

\subsection{Performance in terms of detection probability}

- Let us study the influence of samples number $N$ on detection probability of the FBD-H and the ARGLRT detectors for three different target models (see Table 1 ).

From Figs. 6 and 7, we observe that the FBD-H has a better detection performance compared with the ARGLRT for low values of $N$. In fact, for $N=8$ (cf., Fig. 6), the difference in the signal to clutter ratio (SCR) between these two detectors is greater than $1 \mathrm{~dB}$ for a detection probability equal to 0.5 . This difference becomes less than $1 \mathrm{~dB}$ for $N=16$ (cf., Fig. 7) and as $N$ increases, the two detectors have almost the same detection probability. The advantage of working with low values of $N$ is to ensure the temporal stationarity of the clutter. In fact, previous analysis of recorded real sea clutter data [27,28], showed that this process is non-stationary, both in time and space. 
Table 1

Target models, location of different scatterers and ratio of cells energy with respect to the total energy reflected.

\begin{tabular}{lllllllll}
\hline Target models & \multicolumn{7}{l}{ Cell number } \\
\cline { 2 - 9 } & 1 & 2 & 3 & 4 & 5 & 6 & 7 & 8 \\
\hline$M_{1}$ & $1 / 8$ & $1 / 8$ & $1 / 8$ & $1 / 8$ & $1 / 8$ & $1 / 8$ & $1 / 8$ & $1 / 8$ \\
$M_{2}$ & $5 / 8$ & $2 / 8$ & $1 / 8$ & 0 & 0 & 0 & 0 & 0 \\
$M_{3}$ & 1 & 0 & 0 & 0 & 0 & 0 & 0 & 0
\end{tabular}

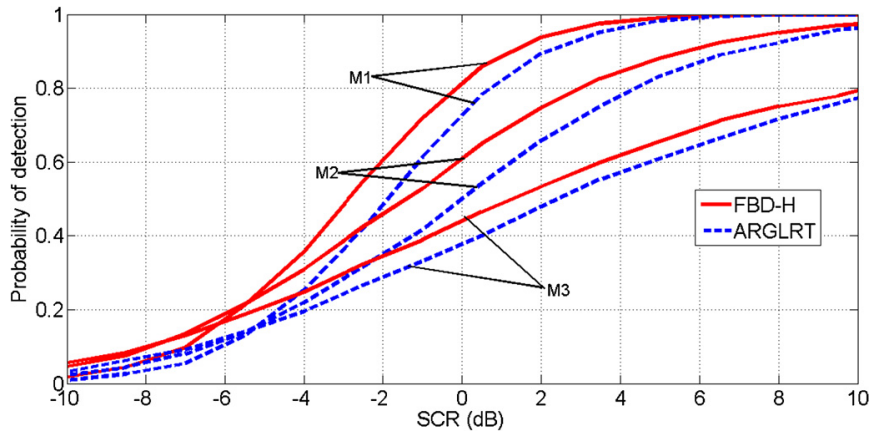

Fig. 6. $P_{\mathrm{d}}$ versus SCR for $N=8$.

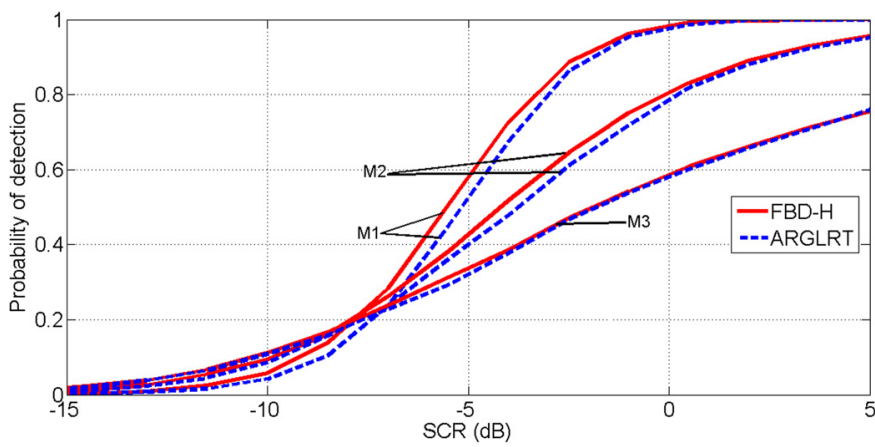

Fig. 7. $P_{\mathrm{d}}$ versus $\mathrm{SCR}$ for $N=16$.

In addition, the aforementioned figures show that the knowledge of the fraction of the cells under test containing the target, is very important (see Table 1).

In the case where the target occupies a fraction of $L$ range cells (e.g., $M_{2}$ and $M_{3}$ models), we observe a degradation in the probability of detection. For example, with the target model $M_{2}$, where the target occupies three range cells with a dominant scatterer in the first range cell, the detection probability of the FBD-H decreases from 0.81 for $M_{1}$ to 0.61 for $M_{2}$ under $\mathrm{SCR}=0 \mathrm{~dB}$ and $N=8$. In the case where the target occupies one range cell, which is given by the target model $M_{3}$, we observe a heavy degradation of the detection probability. This latter decreases to 0.44 for $\mathrm{SCR}=0 \mathrm{~dB}$ and $N=8$, which is due to the fact that one range cell over $L$ cells under test is occupied by the target signal and the rest are occupied by the clutter, decreasing thus the SCR.

- In addition, Fig. 8 shows a comparison between the AR based detectors for $M_{1}$, from which we observe that the proposed FBD-T detector gives better detection probability compared with the FBD-H detector. Specifically, we deduce that the proposed schemes (FBD-T, FBD-H) improve detection performance of the classical scheme, i.e., ARGLRT and ARGLRT-HTG detectors.

- Finally, we study the influence of the number of samples $N$ and $K$ on detection probability of the FBD-H and the AMLGLRT detectors, for a target uniformly distributed among the $L$ range cells. From Fig. 9, we observe that the FBD-H de-

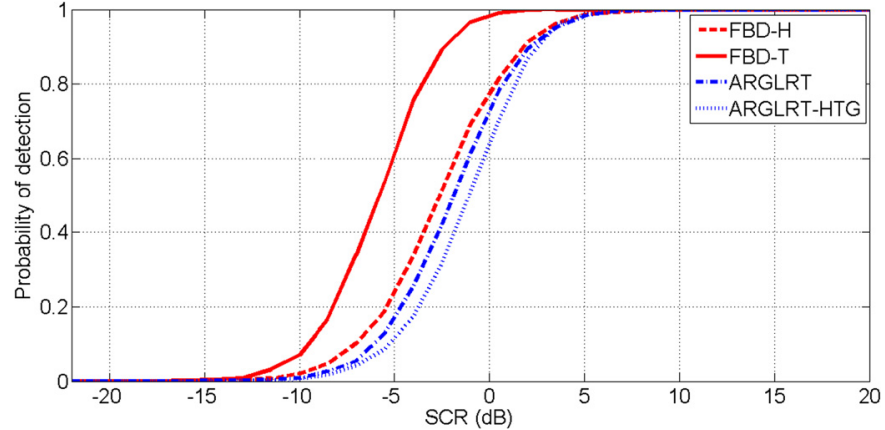

Fig. 8. Comparison between FBD-T, FBD-H, ARGLRT and ARGLRT-HTG for $N=8$ in term of detection probability.

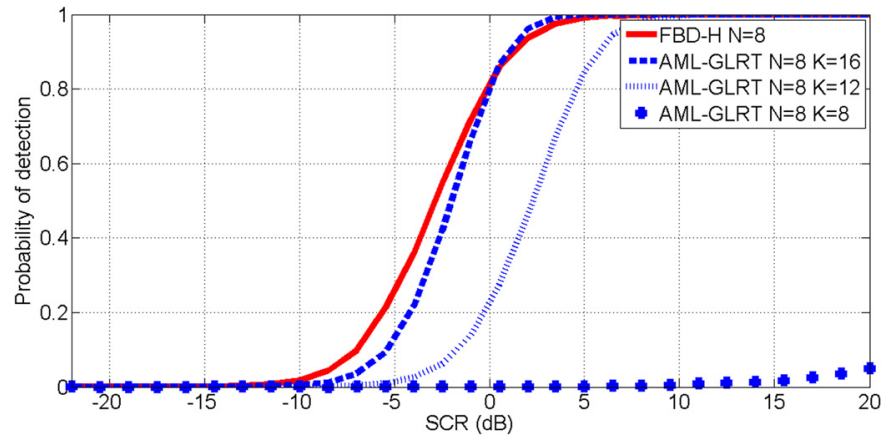

Fig. 9. Comparison between FBD-H and AML-GLRT for $N=8$ and different values of $K$ in term of detection probability.

tector gives better detection performance compared with the AML-GLRT detector for $K \leq 2 N$. Furthermore, we notice that the performance becomes equivalent for $K \gg N$ (not reported here), but in practice it is difficult to obtain a large number of homogeneous secondary data, whereas the FBD-H detector uses only $L$ cells under test, to estimate the clutter covariance matrix and without any secondary data.

\section{Conclusion}

In this paper, we proposed two detectors that require no secondary data, based on both forward and backward auto-regressive GLRT scheme, for which the clutter is modeled by an autoregressive model of order $P$. The proposed FBD-H and FBD-T detectors use the totality of the samples available for the prediction procedure contrary to the classical detectors ARGLRT and ARGLRT-HTG that use only $N-P$ samples. The proposed detectors give better detection performance and have lower false alarm probability compared to the classical detectors ARGLRT and ARGLRT-HTG. Furthermore, it is worth mentioning that they overcome the need for large homogeneous data set, which may be unavailable in practice, contrary to the AML-GLRT detector which requires a number of homogeneous secondary data $K \geq 2 N$ in order to achieve same performance as the proposed detectors.

\section{References}

[1] E. Conte, A.D. Maio, G. Ricci, GLRT-based adaptive detection algorithms for range-spread targets, IEEE Trans. Signal Process. 49 (7) (2001) 1336-1348

[2] D.R. Wehner, High Resolution Radar, vol. 1, Artech House, Inc., Norwood, MA, 1987.

[3] F. Bandiera, D. Orlando, G. Ricci, CFAR detection of extended and multiple point-like targets without assignment of secondary data, IEEE Signal Process. Lett. 13 (4) (2006) 240-243.

[4] K. Gerlach, The effects of signal contamination on two adaptive detectors, IEEE Trans. Aerosp. Electron. Syst. 31 (1) (1995) 297-309. 
[5] A. Farina, F. Studer, R. Vitello, High resolution radar for enhanced target detection, in: Proceedings of the International Conference, Radar 92, vol. 1, 1992, pp. 163-166.

[6] K. Gerlach, M. Steiner, F. Lin, Detection of a spatially distributed target in white noise, IEEE Signal Process. Lett. 4 (7) (1997) 198-200.

[7] K. Gerlach, M. Steiner, Adaptive detection of range distributed targets, IEEE Trans. Signal Process. 47 (7) (1999) 1844-1851.

[8] K. Gerlach, M. Steiner, Fast converging adaptive detection of Doppler-shifted, range-distributed targets, IEEE Trans. Signal Process. 48 (9) (2000) 2686-2690.

[9] E. Conte, A.D. Maio, G. Ricci, CFAR detection of distributed targets in nonGaussian disturbance, IEEE Trans. Aerosp. Electron. Syst. 38 (2) (2002) 612-621.

[10] S. Watts, Special Issue on Radar Clutter and Multipath Propagation, IEE Proceedings, vol. 138, 1991.

[11] F. Gini, A. Farina, Matched subspace CFAR detection of hovering helicopters, IEEE Trans. Aerosp. Electron. Syst. 35 (4) (1999) 1293-1305.

[12] N. Bon, A. Khenchaf, R. Garello, GLRT subspace detection for range and Doppler distributed targets, IEEE Trans. Aerosp. Electron. Syst. 44 (2) (2008) 678-696.

[13] A. Younsi, M. Greco, F. Gini, A. Zoubir, Performance of the adaptive generalised matched subspace constant false alarm rate detector in non-Gaussian noise: an experimental analysis, IET Radar Sonar Navig. 3 (3) (2009) 195-202.

[14] B. Himed, W.L. Melvin, Analyzing space-time adaptive processors using measured data, in: Proc. Thirty-First Asilomar Conf. Signals, Syst., Comput., vol. 1, 1997, pp. 930-935.

[15] S.M. Kay, Asymptotically optimal detection in unknown colored noise via autoregressive modeling, IEEE Trans. Acoust. Speech Signal Process. 31 (4) (1983) 927-940.

[16] A. Sheikhi, M.M. Nayebi, M.R. Aref, Adaptive detection algorithm for radar signals in autoregressive interference, IEE Proc. Radar Sonar Navig. 145 (5) (1998) 309-314.

[17] X. Shuai, L. Kong, J. Yang, AR-model-based adaptive detection of range-spread targets in compound Gaussian clutter, Signal Process. 91 (4) (2011) 750-758.

[18] G. Alfano, A.D. Maio, A. Farina, Model-based adaptive detection of range-spread targets, IEE Proc. Radar Sonar Navig. 151 (1) (2004) 2-10.

[19] F. Gini, M. Greco, Covariance matrix estimation for CFAR detection in correlated heavy tailed clutter, Signal Process. 82 (12) (2002) 1847-1859.

[20] T. Jian, Y. He, F. Su, C. Qu, D. Ping, Adaptive detection of sparsely distributed target in non-Gaussian clutter, IET Radar Sonar Navig. 5 (7) (2011) 780-787.

[21] F. Gini, M. Montanari, L. Verrazzani, Maximum likelihood, ESPRIT, and periodogram frequency estimation of radar signals in K-distributed clutter, Signal Process. 80 (6) (2000) 1115-1126.

[22] S. Haykin, Adaptive Filter Theory, Prentice Hall, 1996.

[23] S. Haykin, A. Steinhardt, Adaptive Radar Detection and Estimation, vol. 2, Wiley-Interscience, 1992.

[24] S. Kay, Fundamentals of Statistical Signal Processing, vol. 2, Prentice Hall, 1998.

[25] K.D. Ward, C.J. Baker, S. Watts, Maritime surveillance radar. Part I: radar scattering from the ocean surface, in: IEE Proc., vol. 137, 1990, pp. 51-62.

[26] X. Shuai, L. Kong, J. Yang, Performance analysis of GLRT-based adaptive detector for distributed targets in compound-Gaussian clutter, Signal Process. 90 (1) (2010) 16-23.

[27] M. Greco, F. Gini, M. Rangaswamy, Statistical analysis of measured polarimetric clutter data at different range resolutions, IEE Proc. Radar Sonar Navig. 153 (2006) 473-481.

[28] M. Greco, F. Gini, A. Younsi, M. Rangaswamy, A. Zoubir, Non-stationary sea clutter: impact on disturbance covariance matrix estimate and detector CFAR, in: International Conference on Radar, 2008, pp. 558-562. 